\title{
A NUMERICAL ALGORITHM FOR FINDING SOLUTIONS OF A GENERALIZED NASH EQUILIBRIUM PROBLEM
}

\author{
LUIZ CARLOS MATIOLI, WILFREDO SOSA, AND JINYUN YUAN
}

\begin{abstract}
A family of nonempty closed convex sets is built by using the data of the Generalized Nash equilibrium problem (GNEP). The sets are selected iteratively such that the intersection of the selected sets contains solutions of the GNEP. The algorithm introduced by Iusem-Sosa (2003) is adapted to obtain solutions of the GNEP. Finally some numerical experiments are given to illustrate the numerical behavior of the algorithm.
\end{abstract}

\section{INTRODUCTION}

The standard definition of a non-cooperative game usually requires that each player in the game has a feasible set (or strategies set) independently on the rival's strategies. In a game with $N$ players, for player $i$-th, we denote by $K_{i} \subset \mathbb{R}^{n_{i}}$ its feasible set and the function $h_{i}: \prod_{i}^{N} K_{i} \rightarrow \mathbb{R}$ will be called the loss function. So, in this game, the feasible set of the game is $\prod_{i}^{N} K_{i}$. The goal of this game is find $\bar{x}$ in the feasible set of the game such that $h_{i}(y) \geq h_{i}(\bar{x}) \forall y \in \prod_{i}^{N} K_{i}$ and $\forall i \in\{1, \cdots, N\}$. It was well understood from the early developments in the field (see $[1,8,10]$ ) that in many cases the interaction among the players can also take place at the feasible set of each player, in this case the feasible set for each player depend on the strategy of the other players, so the feasible set of the game can be different to the product of the feasible set of each player. We speak of Generalized Nash game, when the feasible set of each player depend on the strategies of the other players. Next we shall introduce some notations.

The index set of players is denoted by $I=\{1,2, \ldots, N\}$. For each $x \in \mathbb{R}^{n}$ and each $i \in I, x=\left[x_{i}\right]_{i \in I}$, where $x_{i} \in \mathbb{R}^{n_{i}}$ and $n=\sum_{i \in I} n_{i}$. Now, taking $\Lambda=\prod_{j \in I, j \neq i} \mathbb{R}^{n_{j}}$, we define $x_{-i}=P_{\Lambda}(x)$ where $P_{C}(x)$ denotes the orthogonal projection of $x$ on the set $C$. Note that $x_{i}=P_{\mathbb{R}^{n_{i}}}(x)$. Now, for each $x \in \mathbb{R}^{n}$, each $i \in I$ and each

\footnotetext{
Key words and phrases. Convex feasibility Problem, Generalized Nash Equilibrium Problem, Projection methods.

The work was partially supported by the CNPq, CAPES, and Foundação Araucária, Brazil, CONCYTEC (projects STIC-AMSUD), Peru and Fundación Carolina and CRM, Spain.
} 
$\rho \in \mathbb{R}^{n_{i}}$, we define $x(i, \rho) \in \mathbb{R}^{n}$ as $(x(i, \rho))_{i}=\rho$ and $(x(i, \rho))_{-i}=x_{-i}$. Note, that this notation is different to the classical notation, but we prefer it because we feel it more flexible than the classical one, from the mathematical view point.

For the Generalized Nash game considered here, we denote its feasible set by $K$ and we consider $K$ as a convex closed set. For each $x \in K$, and each $i \in I$, the set $K_{i}(x)=\left\{\rho \in \mathbb{R}^{n_{i}}: x(i, \rho) \in K\right\}$ will be the feasible set of the $i$-th player when the other players choose strategies $x_{-i}$. It is easy to see that

$$
K=\left\{x \in \mathbb{R}^{n}: x_{i} \in K_{i}(x), i=1, \ldots, N\right\} .
$$

For each player $i \in I$, we consider its loss function defined by $h_{i}: \Omega \rightarrow \mathbb{R}$, where $\Omega$ is an open set of $\mathbb{R}^{n}$ and $K \subset \Omega$. Assume that $h_{i}$ is continuously differentiable on $\Omega$ and $h_{i}(x(i, \cdot)): \mathbb{R}^{n_{i}} \rightarrow \mathbb{R} \cup\{+\infty\}$ pseudo-convex (in the sense of Karamardian [6]) for each $x \in K$ and each $i \in I$.

Using the above notation, we state the formal definition of the Generalized Nash Equilibrium Problems (GNEP in the sequel) as follows:

The GNEP is a Nash game, in which the feasible set of each player depends on the other player's strategies, which consists of finding a point in the feasible set of the game $\bar{x} \in K$ such that, for each $i \in I, \bar{x}_{i}$ solves the minimization problem defined by:

$$
\min h_{i}(\bar{x}(i, \rho)) \text { subject to } \rho \in K_{i}(\bar{x}) \text {. }
$$

For recent study of the GNEP, Facchinei and Kanzow have given an excellent survey in [2].

The paper is organized as follows. Some basic results are given In Section 2. A numerical algorithm based on successive projection for solving the GNEP is introduced in Section 3, as a specialization of the other one introduced by Iusem and Sosa ([4]). The convergence of the algorithm is studied as well in the section 3. Compared with other methods, our method is simpler and more efficient. Our method does not require extras variables. Some numerical experiments and comments are given in the last section. In terms of numerical tests, our method can be applied to solve large scale problems.

\section{Previous Results}

Given the GNEP, we define:

$$
\begin{gathered}
F: \Omega \rightarrow \mathbb{R}^{n} \text { by } F(x)=\left[\nabla_{i} h_{i}(x(i, \cdot))\left(x_{i}\right)\right]_{i \in I} \\
f: \Omega \times \mathbb{R}^{n} \rightarrow \mathbb{R} \text { by } f(x, y)=\langle F(x), x-y\rangle
\end{gathered}
$$

for each $x \in K$ :

$$
L_{f}(x)=\{y \in K: f(x, y) \geq 0\}
$$

Remark 2.1. We point out that $f(x, y)=\sum_{i \in I}\left\langle\nabla_{i} h_{i}(x(i, \cdot))\left(x_{i}\right), x_{i}-y_{i}\right\rangle$ $\forall x, y \in K$. So, when $y=x(i, \rho)$ for $i \in I$ and $\rho \in K_{i}$, then $f(x, y)=$ $\left\langle\nabla_{i} h_{i}(x(i, \cdot))\left(x_{i}\right), x_{i}-\rho\right\rangle$, because for each $j \neq i, y_{j}=x_{j}$ and so $x_{j}-y_{j}=0$. 
The next lemma follows directly from the previous definitions without proof.

Lemma 2.2. The following statements hold.

(1) The function $f$ is continuous on $\Omega \times \mathbb{R}^{n}$.

(2) For each $x \in \Omega, f(x, x)=0$.

(3) For each $x \in \Omega, f(x, \cdot): \mathbb{R}^{n} \rightarrow \mathbb{R}$ is linear affine (in particular is convex and concave).

(4) For each $y \in \Omega, L_{f}(y)$ is nonempty closed and convex (in particular if $K$ is a polyhedron, then $L_{f}(y)$ is also a polyhedron).

Lemma 2.3. If $x^{*} \in K$ is a local minimal point of the function $f\left(\cdot, x^{*}\right): K \rightarrow \mathbb{R}$, then $x^{*}$ is a solution of GNEP.

Proof. If $x^{*} \in K$ is local minimal point of the function $f\left(\cdot, x^{*}\right): K \rightarrow \mathbb{R}$, then there exists a nonempty open convex set $U$ such that $x^{*} \in U \cap K$ and $0=f\left(x^{*}, x^{*}\right) \leq f\left(y, x^{*}\right)$ for each $y \in U \cap K$. Now, $\forall y \in K$ and $y \neq x^{*}$, there exists $\bar{t} \in] 0,1\left[\right.$ such that $x_{t}=t y+(1-t) x^{*} \in U \cap K$ for each $\left.t \in\right] 0, \bar{t}[$. But, $0=f\left(x_{t}, x_{t}\right)=t f\left(x_{t}, y\right)+(1-t) f\left(x_{t}, x^{*}\right)$ for each $\left.t \in\right] 0, \bar{t}$. Thus, $f\left(x_{t}, y\right) \leq 0$ for each $t \in] 0, \bar{t}\left[\right.$. In the limit, we have that $f\left(x^{*}, y\right) \leq 0$. Now, for each $i \in I, \rho \in K_{i}\left(x^{*}\right)$ and $y=x^{*}(i, \rho)$, we have, from the previous remark, that $\left\langle\nabla_{i} h_{i}\left(x^{*}\right), \rho-x_{i}^{*}\right\rangle=-f\left(x^{*}, y\right) \geq 0$. Therefore, the statement follows from the pseudo-convexity of the functions $h_{i}$ for each $i \in I$.

Lemma 2.4. If there exists $x^{*} \in K$ such that $F\left(x^{*}\right)=0$, then $x^{*}$ is a solution of GNEP.

Proof. Follows from the pseudo convexity of the functions $h_{i}$ for each $i \in I$.

\section{The Algorithm}

In this section, we introduce an algorithm, based on a successive projection scheme. Each iteration basically consists of two steps: an inexact local minimization of a continuous function over a compact set, and an orthogonal projection onto a set of the form $L_{f}(y)$ for some $y \in K$. The algorithm requires a constant $\alpha \in(0,1)$ and three parameter sequences: relaxation parameters $\left\{\lambda_{m}\right\} \subset[\alpha, 2-\alpha]$, precision parameters $\left\{\varepsilon_{m}>0\right\} \downarrow 0$ for the inexact maximization, and $\left\{\delta_{m}>\alpha\right\} \uparrow \delta$ for the local minimization respectively. The algorithm generates two sequences $\left\{x^{m}\right\},\left\{y^{m}\right\} \subset K$ in the following way.

\section{Algorithm 3.1.}

Initial step: Choose $x^{0} \in K$.

Iterative step: Given $x^{m} \in K$,

a) Find $y^{m} \in K \cap \bar{B}\left(x^{m}, \delta_{m}\right)$ satisfying

$$
f\left(y^{m}, x^{m}\right) \leq \min \left\{f\left(y, x^{m}\right): y \in K \cap \bar{B}\left(x^{m}, \delta_{m}\right)\right\}+\varepsilon_{m} .
$$


b) Compute $x^{m+1} \in K$ as

$$
x^{m+1}=x^{m}+\lambda_{m}\left(P_{L_{f}\left(y^{m}\right)}\left(x^{m}\right)-x^{m}\right) .
$$

Next we shall study the properties of the sequences generated by Algorithm 3.1.

Lemma 3.1. Given a convergent sequence $\left\{x^{m}\right\} \subset \mathbb{R}^{n}$ and $\left\{\delta_{m}>\alpha\right\} \uparrow \delta$. If $x^{*}$ is the limit of the sequence $\left\{x^{m}\right\}$, then for each $\epsilon>0$, there exists $M>0$ such that for all $m \geq M$, the following statements hold.

(1) $\left\|x^{m}-x^{*}\right\|<\frac{\epsilon}{2}$.

(2) $\delta_{m}>\delta-\frac{\epsilon}{2}$.

(3) $B\left(x^{*}, \delta-\epsilon\right) \subset B\left(x^{m}, \delta_{m}\right) \subset B\left(x^{*}, \delta+\frac{\epsilon}{2}\right)$.

(4) For all $y \in B\left(x^{*}, \delta\right)$, there exists $i \geq m$ such that $\delta_{i} \geq\left\|y-x^{i}\right\|$

Proof. Since $\left\{x^{m}\right\}$ converges to $x^{*}$, the sequence $\left\{\left\|x^{m}-x^{*}\right\|\right\}$ converges to zero. Hence, there exists $M_{1}>0$ such that $\left\|x^{m}-x^{*}\right\|<\frac{\epsilon}{2}$ for each $m \geq M_{1}$. Also since $\left\{\delta_{m}\right\} \uparrow \delta=\sup \left\{\delta_{m}: m \in \mathbb{N}\right\}$, there exists $M_{2}>0$ such that $\delta_{m}>\delta-\frac{\epsilon}{2}$. Then, items (1) and (2) follows from taking $M=\max \left\{M_{1}, M_{2}\right\}$. Now, for $y \in B\left(x^{*}, \delta-\epsilon\right),\left\|y-x^{m}\right\| \leq\left\|y-x^{*}\right\|+\left\|x^{*}-x^{m}\right\| \leq \delta-\epsilon+\frac{\epsilon}{2}=\delta-\frac{\epsilon}{2}<\delta_{m}$. The other inclusions are analogous. Thus item (3) follows. For item (4), suppose that there exists $m \geq M$ and $y \in B\left(x^{*}, \delta\right)$ such that $\delta_{i}<\left\|y-x^{i}\right\|$ for all $i \geq m$. Taking the limit, we obtain $\delta \leq\left\|y-x^{*}\right\|$ which is a contradiction because $y \in B\left(x^{*}, \delta\right)$. The proof is complete.

Now, we start the convergence analysis of the algorithm

Proposition 3.2. Algorithm 3.1 is well defined.

Proof. Since $x^{m} \in K$ for all $m$, all intersections $K \cap \bar{B}\left(x^{m}, \delta_{m}\right)$ are nonempty and trivially compact. It follows from the continuity of $f$ that $f\left(\cdot, x^{m}\right)$ attains its minimum over $K \cap \bar{B}\left(x^{m}, \delta_{m}\right)$. Hence, there exists $y^{m}$ satisfying (6). Since, $L_{f}\left(y^{m}\right)$ is closed and convex, $x^{m+1}$ is uniquely defined by $(7)$.

Lemma 3.3. Let $\left\{x^{m}\right\}$ and $\left\{y^{m}\right\}$ be the sequences generated by Algorithm 3.1. If there exists $M>0$ such that $\bigcap_{m \geq M} L_{f}\left(y^{m}\right)$ is nonempty, then

(1) The sequence $\left\{\left\|x^{m}-\bar{x}\right\|\right\}$ is convergent, for each $\bar{x} \in \bigcap_{m \geq M} L_{f}\left(y^{m}\right)$.

(2) The sequence $\left\{x^{m}-P_{L_{f}\left(y^{m}\right)}\left(x^{m}\right)\right\}$ converges to zero.

(3) The sequences $\left\{x^{m}\right\}$ and $\left\{y^{m}\right\}$ are bounded.

Proof. $\quad$ (1) Set $\bar{x} \in \bigcap_{m \geq M} L_{f}\left(y^{m}\right)$, by (7):

$$
\begin{gathered}
\left\|x^{m+1}-\bar{x}\right\|^{2}=\left\|x^{m}-\bar{x}\right\|^{2}+\lambda_{m}^{2}\left\|x^{m}-P_{L_{f}\left(y^{m}\right)}\left(x^{m}\right)\right\|^{2} \\
\quad+2 \lambda_{m}\left\langle x^{m}-\bar{x}, P_{L_{f}\left(y^{m}\right)}\left(x^{m}\right)-x^{m}\right\rangle \\
=\left\|x^{m}-\bar{x}\right\|^{2}+\lambda_{m}^{2}\left\|x^{m}-P_{L_{f}\left(y^{m}\right)}\left(x^{m}\right)\right\|^{2}-2 \lambda_{m}\left\|x^{m}-P_{L_{f}\left(y^{m}\right)}\left(x^{m}\right)\right\|^{2} \\
\quad+2 \lambda_{m}\left\langle P_{L_{f}\left(y^{m}\right)}\left(x^{m}\right)-\bar{x}, P_{L_{f}\left(y^{m}\right)}\left(x^{m}\right)-x^{m}\right\rangle .
\end{gathered}
$$


Since $\bar{x}$ belongs to $L_{f}\left(y^{m}\right)$ for each $m \geq M$, the last term in the previous expression is nonnegative, because of the following well known property of orthogonal projections: $\left\langle P_{C}(x)-y, P_{C}(x)-x\right\rangle \leq 0$ for any closed and convex set $C$, any $x \in \mathbb{R}^{n}$ and any $y \in C$. Thus, it follows that, for all $m \geq M$,

$$
\left\|x^{m+1}-\bar{x}\right\|^{2} \leq\left\|x^{m}-\bar{x}\right\|^{2}-\lambda_{m}\left(2-\lambda_{m}\right)\left\|x^{m}-P_{L_{f}\left(y^{m}\right)}\right\|^{2} \leq\left\|x^{m}-\bar{x}\right\|^{2},
$$

by the fact that $\lambda_{m}\left(2-\lambda_{m}\right)>0$. We conclude from (8) that the sequence $\left\{\left\|x^{m}-\bar{x}\right\|\right\}_{m \geq M}$ is non-increasing, and henceforth, being nonnegative, so $\left\{\left\|x^{m}-\bar{x}\right\|\right\}_{m=0}^{\infty}$ is convergent.

(2) Rewriting (8) and using the conditions on $\left\{\lambda_{m}\right\}$ we get

$$
\begin{aligned}
\alpha(2-\alpha)\left\|x^{m}-P_{L_{f}\left(y^{m}\right)}\left(x^{m}\right)\right\|^{2} & \leq \lambda_{m}\left(2-\lambda_{m}\right)\left\|x^{m}-P_{L_{f}\left(y^{m}\right)}\left(x^{m}\right)\right\|^{2} \\
& \leq\left\|x^{m}-\bar{x}\right\|^{2}-\left\|x^{m+1}-\bar{x}\right\|^{2} .
\end{aligned}
$$

using the convergence of $\left\{\left\|x^{m}-\bar{x}\right\|\right\}$ we obtain, since $\alpha(2-\alpha)>0$,

$$
\lim _{m \rightarrow \infty}\left(x^{m}-P_{L_{f}\left(y^{m}\right)}\left(x^{m}\right)\right)=0 .
$$

(3) The convergence of $\left\{\left\|x^{m}-\bar{x}\right\|\right\}$ also implies that the sequence $\left\{x^{m}\right\}$ is bounded. For each $m$, since $y^{m} \in K \cap \bar{B}\left(x^{m}, \delta_{m}\right),\left\|y^{m}\right\|-\left\|x^{m}\right\| \leq$ $\left\|y^{m}-x^{m}\right\| \leq \delta_{m}$. Thus, $\left\|y^{m}\right\| \leq\left\|x^{m}\right\|+\delta_{m}$. The statement follows from the facts that $\left\{\left\|x^{m}\right\|\right\}$ and $\left\{\delta_{m}\right\}$ are bounded.

Let $\left\{x^{m}\right\}$ be sequence generated by Algorithm 3.1. Remember that $x^{*}$ is a cluster point of $\left\{x^{m}\right\}$ if there is a subsequence $\left\{x^{m_{i}}\right\}$ of $\left\{x^{m}\right\}$ such that $\left\{x^{m_{i}}\right\}$ converges to $x^{*}$.

Theorem 3.4. Let $\left\{x^{m}\right\}$ and $\left\{y^{m}\right\}$ be the sequences generated by Algorithm 3.1 and let $M>0$.

i) If $\bigcap_{m>M} L_{f}\left(y^{m}\right)$ is nonempty, then any cluster point of $\left\{x^{m}\right\}$ is a local minimizer of $f\left(\cdot, x^{*}\right)$ over $K$.

ii) If $\left\{x^{m}\right\}$ converges to $x^{*} \in K$, then $x^{*}$ is local minimizer of $f\left(\cdot, x^{*}\right)$ over $K$.

iii) If GNEP lacks solutions, then $\left\{x^{m}\right\}$ is not convergent (though it might be bounded).

Proof. $\quad$ i) From Lemma 3.3, we know that $\left\{x^{m}\right\}$ and $\left\{y^{m}\right\}$ are bounded. Let $x^{*}$ be a cluster point of $\left\{x^{m}\right\} \subset K$. Since $K$ is closed, $x^{*}$ belongs to $K$. Thus, we can select a subsequence $\left\{x^{k_{m}}\right\}$ of $\left\{x^{m}\right\}$ such that $\lim _{m \rightarrow \infty} x^{k_{m}}=$ $x^{*}$ and $\left\{y^{k_{m}}\right\}$ converges to $y^{*}$. By $(9), \lim _{m \rightarrow \infty} P_{L_{f}\left(y^{k_{m}}\right)}\left(x^{k_{m}}\right)=x^{*}$. It follows from the continuity of $f$ that

$$
f\left(y^{*}, x^{*}\right)=\lim _{m \rightarrow \infty}\left(f\left(y^{k_{m}}, P_{L_{f}\left(y^{k_{m}}\right)}\left(x^{k_{m}}\right)\right) \geq 0,\right.
$$


where the inequality follows from the definition of $L_{f}\left(y^{k_{m}}\right)$ and the fact that

$$
P_{L_{f}\left(y^{k_{m}}\right)}\left(x^{k_{m}}\right) \in L_{f}\left(y^{k_{m}}\right) .
$$

From Item (3) of Lemma 3.1, for any fixed $\epsilon>0$, there exists $M_{\epsilon}>0$ such that $B\left(x^{*}, \delta-\epsilon\right) \subset B\left(x^{k_{m}}, \delta_{k_{m}}\right)$ for all $k_{m}>M_{\epsilon}$. Taking $\bar{M}=$ $\max \left\{M, M_{\epsilon}\right\}$, for each $y \in B\left(x^{*}, \delta-\epsilon\right)$ and each $k_{m}>\bar{M}$, we obtain from equation (6) of Algorithm 3.1 that $f\left(y^{k_{m}}, x^{k_{m}}\right) \leq f\left(y, x^{k_{m}}\right)+\epsilon_{k_{m}}$. Hence, in the limit, $f\left(x^{*}, x^{*}\right)=0 \leq f\left(y^{*}, x^{*}\right) \leq f\left(y, x^{*}\right)$ which implies that $x^{*}$ is a local minimum of $f\left(\cdot, x^{*}\right): K \rightarrow \mathbb{R}$.

ii) If $\left\{x^{m}\right\}$ converges to $x^{*}$, then $\left\{x^{m}\right\}$ and $\left\{y^{m}\right\}$ are bounded and $\lim _{m \rightarrow \infty} P_{L_{f}\left(y^{k_{m}}\right)}\left(x^{k_{m}}\right)=x^{*}$. Thus, the statement follows from the same argument as in the previous item.

iii) Suppose that $\left\{x^{m}\right\}$ converges to $x^{*}$. From the previous item, $x^{*}$ is a local minimum of $f\left(\cdot, x^{*}\right)$. From Lemma $2.3 x^{*}$ is a solution of the GNEP which contradicts to the hypothesis of this item..

Next we shall consider the Convex Feasibility Problem (CFP in short)

$$
\cap_{y \in K} L_{f}(y)
$$

which is associated to GNEP.

Corollary 3.5. If the CFP has solutions, then every cluster point of the sequence $\left\{x^{m}\right\}$ generated by Algorithm 3.1 is a solution of the GNEP.

Proof. If the CFP has solutions, then $\emptyset \neq \cap_{y \in K} L_{f}(y) \subset \cap_{m=1}^{\infty} L_{f}\left(y^{m}\right)$. The result follows from Theorem 3.4(i) immediately.

In the next result we consider the notion of pseudomonotonicity (for details see for instance [6], [5]) for the operator $F$ defined in 3, i.e. $F$ is pseudomonotone if for $x, y \in \operatorname{dom}(T):\langle F(x), y-x\rangle \geq 0$ implies $\langle F(y), x-y\rangle \leq 0$. Note that the previous implication is equivalent to say that $\langle F(x), y-x\rangle>0$ implies $\langle F(y), x-y\rangle<0$.

Lemma 3.6. If $F$ is pseudomonotone, then $\left\{L_{f}(y)\right\}_{y \in K}$ is such that for each $\left\{x^{1}, \cdots, x^{p}\right\} \subset K$ we have that $\operatorname{co}\left\{x^{1}, \cdots, x^{p}\right\} \subset \bigcup_{i=1}^{p} L_{f}\left(x^{i}\right)$

Proof. Suppose that $\exists \bar{x} \in \operatorname{co}\left\{x^{1}, \cdots, x^{p}\right\}$ such that $\bar{x} \notin \bigcup_{i=1}^{p} L_{f}\left(x^{i}\right)$, then $\bar{x}=$ $\sum_{i=1}^{p} \lambda_{i} x^{i}$ with $\lambda_{i} \geq 0$ and $\sum_{i=1}^{p} \lambda_{i}=1$ and $\left\langle F\left(x^{i}\right), x^{i}-\bar{x}\right\rangle<0, \forall i$, then $\left\langle F\left(x^{i}\right), \bar{x}-x^{i}\right\rangle>0, \forall i$, So, from the pseudomonotonicity of $F$, we have that $\left\langle F(\bar{x}), x^{i}-\bar{x}\right\rangle<0, \forall i$. So, $0=\langle F(\bar{x}), \bar{x}-\bar{x}\rangle=\sum_{i=1}^{p} \lambda_{i}\left\langle F(\bar{x}), x^{i}-\bar{x}\right\rangle<0$. The statement follows from this contradiction.

Corollary 3.7. Let $\left\{x^{m}\right\}$ and $\left\{y^{m}\right\}$ be the sequences generated by Algorithm 3.1. If $F$ is pseudo-monotone and either $\left\{x^{m}\right\}$ or $\left\{y^{m}\right\}$ is bounded, then every cluster point of the sequence $\left\{x^{m}\right\}$ is a solution of the GNEP. 
Proof. If $\left\{x^{m}\right\}$ is bounded, then $\left\{y^{m}\right\}$ is bounded by the same argument as in the proof of (i) of Theorem 3.4. Thus we may assume that $\left\{y^{m}\right\}$ is bounded. By the previous Lemma and Lemma 2.3 in $[4] \cap_{m=1}^{\infty} L_{f}\left(y^{m}\right) \neq \emptyset$. The statement follows from (i) of Theorem 3.4.

\section{NumericAl EXPERIMENTS}

First of all we set $K=\left\{x \in \mathbb{R}^{n}:\left\langle a^{i}, x\right\rangle \leq b_{i}, x_{j} \geq 0, i=1, \ldots, m, j=1, \ldots, n\right\}$ as a polyhedron

In (6) we minimize a linear approximation of $f$ in a compact set, that is, we solve the following subproblem: (given $x^{m}$ in $K$ and $\delta^{m}$ )

$$
\begin{array}{cc}
\text { minimize } & \left\langle F\left(x^{m}\right), y-x^{m}\right\rangle \\
\text { s.t. } & y \in \bar{B}\left(x^{m}, \delta^{m}\right) \cap K
\end{array}
$$

to find $y^{m}$. Note that in our implementation the region $\bar{B}\left(x^{m}, \delta^{m}\right)$ is a box.

In step (b) of Algorithm 3.1, we determine $x^{m+1}$ by an orthogonal projection of $x^{m}$ onto $L_{f}\left(y^{m}\right)$, which is equivalent to solving the following subproblem:

$$
\begin{array}{cl}
\text { minimize } & \frac{1}{2}\left\|z-x^{k}\right\|^{2} \\
\text { s.t. } & z \in K \cap\left\{y:\left\langle F\left(x^{m}\right), y\right\rangle \leq\left\langle F\left(x^{m}\right), x^{m}\right\rangle\right\}
\end{array}
$$

The subproblems (11) end (12) are solved, respectively, by routines linprog end lsqlin from optimization toolbox of Matlab 6.0.0.88 (R12). The stop criteria is $\frac{\left\|x^{m+1}-x^{m}\right\|}{\left\|x^{m+1}\right\|}<10^{-10}$.

The initial point in $K$ is determined by solving

$$
\min _{s, x} \sum_{i=1}^{m} s_{i}
$$

subject to $A x+s=b, s, x \geq 0$.

We consider only nonnegative $x$ because negative variables have no real application meaning for most practical problems. In the implementation of Algorithm 3.1 we initialize $\alpha=0.2$ in Problem 2 , and $\alpha=0.5$ in all other problems. The parameter $\delta_{m}$ controls the radio of box $\bar{B}\left(x^{m}, \delta^{m}\right)$ and is increased till $\delta_{\max }$. The updating form of this parameter is given by $\delta_{0}=\left(\alpha+\delta_{\max }\right) / 2$ and $\delta_{m+1}=\left(\delta_{m}+\delta_{\max }\right) / 2$. All computations are executed by Matlab 6.0.0.88 on Laptop TOSHIBA Satellite M115-S3144 with intel Core 2 with 2 GB de RAM Memory.

Problem 1: $F\left(x_{1}, x_{2}\right)=\left(3 x_{1}^{2} x_{2}^{2}, 3 x_{1}^{2} x_{2}^{2}\right)$ and $K=\left\{\left(x_{1}, x_{2}\right): x_{1}+x_{2} \leq 3\right.$, $\left.x_{1} \geq 1 ; x_{2} \geq 1\right\}$. The exact solution is $x=\left[\begin{array}{ll}1 & 1\end{array}\right]^{T}$. The numerical results are showed in Table 1, whose first column is the number of iterations, the second and third columns are the decision of players. 
Problem 2: This problem is taken from [3] where authors transform the Nash equilibrium problem to Variational Inequality. In sense of Variational Inequality the solution is unique $\bar{x}=\left(\frac{3}{4}, \frac{1}{4}\right)$ which belongs to solution set of the Nash equilibrium problem. Here the problem is given by: $F\left(x_{1}, x_{2}\right)=\left(2 x_{1}-2,2 x_{2}-1\right)$ and $K=\left\{\left(x_{1}, x_{2}\right): x_{1}+x_{2} \leq 1, x_{1} \geq 0, x_{2} \geq 0\right\}$.

\begin{tabular}{|c|c|c|}
\hline $\mathrm{m}$ & $x_{1}^{m}$ & $x_{2}^{m}$ \\
\hline \hline 0 & 1.3618 & 1.3618 \\
\hline 1 & 1.0618 & 1.0618 \\
\hline 2 & 1.0000 & 1.0000 \\
\hline
\end{tabular}

TABle 1. Numerical results for Problem 1

Problem 3: We consider the River Basin Pollution problem [7] with three players $j=1,2,3$. The problem is given by $h_{j}(x)=p_{j} x_{j}^{2}+0.01 x_{j}\left(x_{1}+x_{2}+x_{3}\right)-q_{j} x_{j}$. Now using $F(x)=\left[\nabla_{j} h_{j}(x(j,)).\left(x_{j}\right)\right], j=1,2,3$, we have

$$
F(x)=\left[\begin{array}{l}
2 p_{1} x_{1}+0.01\left(2 x_{1}+x_{2}+x_{3}\right)-q_{1} \\
2 p_{2} x_{2}+0.01\left(x_{1}+2 x_{2}+x_{3}\right)-q_{2} \\
2 p_{3} x_{3}+0.01\left(x_{1}+x_{2}+2 x_{3}\right)-q_{3}
\end{array}\right]
$$

where $p=[0.01 ; 0.05 ; 0.01]^{T}$ and $q=[2.9 ; 2.88 ; 2.85]^{T}$. The polyhedron $K$ is given by constraints: $g_{1}(x)=3.25 x_{1}+1.25 x_{2}+4.125 x_{3} \leq 100, g_{2}(x)=2.2915 x_{1}+$ $1.5625 x_{2}+2.8125 x_{3} \leq 100$, and $x_{1}, x_{2}, x_{3} \geq 0$.

Problema 4[9]: The authors solve the problem by means of Linear Complementary Problem whose solution depends on multipliers. The problem is defined by $F(x)=\left(F_{1}(x), F_{2}(x)\right)^{T}$ where $F_{1}(x)=-1+x_{1}+0.5 x_{2}$ and $F_{2}(x)=-2+0.5 x_{1}+x_{2}$. Here the polyhedron is given by $x_{1}+x_{2} \leq 1, x_{1}, x_{2} \geq 0$. The solution founded by our algorithm is $\bar{x}=\left(x_{1}, x_{2}\right)=(0,1)$.

Problem 5 [7]: The problem is give by

$$
F(x)=\left[\frac{2\left(x_{1}+x_{2}\right)}{4}+\frac{2\left(x_{1}-x_{2}\right)}{9} ; \frac{2\left(x_{1}+x_{2}\right)}{4}-\frac{2\left(x_{1}-x_{2}\right)}{9}\right] .
$$

Here $K=\left\{0 \leq x_{1}, x_{2} \leq 10\right\}$.

Problems 6-10. We define the problems by

$$
F_{j}(x)=2 p_{j} x_{j}+\alpha x_{j}+\alpha \sum_{k=1}^{n} x_{k}-q_{j}
$$


where $F_{j}(x)$ is the $j$-th component of $F(x)$, the constants $p, \alpha$ as well as the $m$ constraints are generated by function rand of Matlab with corresponding dimensions given in Table 2. The purpose of these problems is to test the numerical behavior of Algorithm 3.1 for large scale problems.

The numerical results and comparisons are given in Table 2 where the first column is the problem, second column is the number of variable, third column is the number of constraints, forth column is the parameter $\alpha$, fifth column is the $\delta_{\text {max }}$, sixth column is number of iteration, seventh column is the number of the iterations of the method given in the cited references. Here - means that there is no number of iteration for other methods.

\begin{tabular}{|c|c|c|c|c|c||c|}
\hline Problem & $\mathrm{n}$ & $\mathrm{m}$ & $\alpha$ & $\delta_{\max }$ & nit & IT of other methods \\
\hline \hline Prob2 & 2 & 1 & 0.2 & 0.3 & 2 & $-[3]$ \\
\hline Prob3 & 2 & 2 & 0.5 & 1.3 & 9 & $7[7]$ \\
\hline Prob4 & 2 & 1 & 0.5 & 1.3 & 2 & $-[9]$ \\
\hline Prob5 & 3 & 2 & 0.5 & 1.5 & 15 & $19[7]$ \\
\hline Prob6 & 50 & 30 & 0.5 & 1 & 3 & - \\
\hline Prob7 & 100 & 80 & 0.5 & 1 & 4 & - \\
\hline Prob8 & 200 & 120 & 0.5 & 1 & 3 & - \\
\hline Prob9 & 500 & 50 & 0.5 & 1 & 5 & - \\
\hline Prob10 & 1000 & 30 & 0.5 & 1 & 2 & - \\
\hline
\end{tabular}

TABLE 2. Numerical results and comparisons

The main advantages of Algorithm 3.1 compared with other methods are as follows.

(1) Algorithm 3.1 doesn't require extra variables unlike other methods given in $[7,9]$.

(2) By our numerical results, it seems that the convergence of Algorithm 3.1 is independent on number of variables which will be studied in the future.

(3) The convergence rate is very competitive from our numerical experiments.

(4) So far Algorithm 3.1 is the best compared with all existing methods for the problem.

We shall give further study and modifications of Algorithm 3.1 in the next paper.

\section{ACKNOWLEDGMENT}

This work was started during the visit of the second author at Department of Mathematics, Federal University of Paraná in 2008 and was finished during the 
visit of the same author at Centre de Recerca Matematica (CRM) in 2009. The second author likes to thank Prof. Carlos Henrique dos Santos, Prof. Jinyun Yuan and Prof. Juan Enrique Martinez Legaz for their kindest hospitality during his visiting at: Department of Mathematics, Federal University of Paraná, Curitiba, Brazil and the CRM of Barcelona, Spain; respectively.

\section{REFERENCES}

[1] K.J. Arrow, G. Debreu. Existence of an equilibrium for a competitive economy, Econometrica 22 (1954), 265-290.

[2] F. Facchinei, C. Kanzow. Generalized Nash equilibrium problems 4OR 5 (2007), 173-210.

[3] F. Facchinei, A. Fischer, V. Piccialli. On generalized Nash games and variational inequalities Operations Research Letters 35 (2007), 159-164.

[4] A.N. Iusem, W. Sosa. Iterative algorithms for equilibrium problems, Optimization $\mathbf{5 2}$ (2003,) 301-316.

[5] A.N. Iusem, W. Sosa. New existence results for equilibrium problems, Nonlinear Analysis 52 (2003), 621-635.

[6] S. Karamardian, Complementarity problems over cones with monotone and pseudomonotone maps, J. Optimization Theory Appl., 18(4) (1976), 445-454.

[7] J.B. Krawczyk, S. Uryasev. relaxations algorithms to find Nash equilibria with economic applications, Environmental Modeling and Assessment 5 (2000), 63-73.

[8] L.W. McKenzie. On the existence of a general equilibrium for a competitive market, Econometrica 27 (1959), 54-71.

[9] J.S Pang, B.F. Hobbs. Nash-Cournot equilibria in electric power markets with piecewise linear demand functions and joint constraints Operations Research 55, issue 1 (january 2007) 113-127.

[10] J.B. Rosen. Existence and uniqueness of equilibrium points for concave $n$-person games, Econometrica 33 (1965), 520-534.

Luiz Carlos Matioli

Departamento de Matemática - UfPr Centro Politénico

CP: 19.081 , CURITIBA

PR 81531-980, BRAZIL

E-mail address: matioli@mat.ufpr.br

WiLFREDO SOSA

Instituto de Matemática y Ciencias Afines

Universidad NACIONAL DE INGENIERIA

Calle los Biologos 245, Lima 12

Lima Perú

E-mail address: sosa@uni.edu.pe

JINYUN YUAN

Departamento de Matemática - UfPR, Centro Politénico

CP: 19.081, CURITIBA

PR, 81531-980,BRAZIL

E-mail address: jin@mat.ufpr.br 